\title{
Effects of step duration in incremental ramp protocols on peak power and maximal oxygen consumption
}

\author{
Alessandra Adami • Andrea Sivieri • \\ Christian Moia $\cdot$ Renza Perini $\cdot$ Guido Ferretti
}

Received: 24 April 2013/Accepted: 29 July 2013/Published online: 15 August 2013

(C) Springer-Verlag Berlin Heidelberg 2013

\begin{abstract}
Purpose Morton (J Sport Sci 29:307-309, 2011) proposed a model of the peak power attained in ramp protocol $\left(\dot{w}_{\text {peak }}\right)$ that included critical power $(\mathrm{CP})$ and anaerobic capacity as constants, and mean ramp slope $(S)$ as variable. Our hypothesis is that $\dot{w}_{\text {peak }}$ depends only on $S$, so that Morton's model should be applicable in all types of ramps. The aim of this study was to test this hypothesis by validating Morton's model using stepwise ramp tests with invariant step increment and increasing step duration.

Methods Sixteen men performed six ramp tests with $25 \mathrm{~W}$ increments. Step duration was: 15, 30, 60, 90, 120 and $180 \mathrm{~s}$. Maximal oxygen consumption $\left(\dot{V} \mathrm{O}_{2 \max }\right)$ and $\dot{w}_{\text {peak }}$ were identified as the highest values reached during each test. An Åstrand-type test was also performed. We measured oxygen consumption and ventilatory variables, together with lactate and heart rate.

Results $\quad \dot{V} \mathrm{O}_{2 \max }$ was the same in all tests; $\dot{w}_{\text {peak }}$ was significantly lower the longer the step duration, and all values differed from the maximal power of the Åstrand-type test $\left(\dot{w}_{\max }\right)$. Morton's model yielded an excellent fitting, with mean CP equal to $198.08 \pm 37.46 \mathrm{~W}$ and anaerobic capacity equal to $16.82 \pm 5.69 \mathrm{~kJ}$.
\end{abstract}

Communicated by Jean-René Lacour.

A. Adami $(\bowtie) \cdot$ C. Moia $\cdot$ G. Ferretti

Département de Neuroscience Fondamentales, Centre Médical

Universitaire, Université de Genève, 1 Rue Michel Servet,

1211 Genève 4, Switzerland

e-mail: alessandra.adami@unige.ch; adami.alsn@gmail.com

A. Sivieri · R. Perini · G. Ferretti

Dipartimento di Scienze Cliniche E Sperimentali, Università di

Brescia, Viale Europa 11, 25123 Brescia, Italy
Conclusions Morton's model is a good descriptor of the mechanics of ramp tests. Further developments of Morton's model demonstrated that, whereas $\dot{w}_{\text {peak }}$ is a protocoldependent variable, the difference between $\dot{w}_{\max }$ and CP is a constant, so that their values do not depend on the protocol applied.

Keywords Critical power - Maximal aerobic power . Incremental test · Morton's model · Anaerobic capacity

$\begin{array}{ll}\text { Abbreviations } \\ a & \text { Whipp's model constant equal to anaerobic work } \\ b & \text { Whipp's model constant equal to maximal } \\ & \text { mechanical aerobic power } \\ \text { CP } & \text { Critical power } \\ \text { HR } & \text { Heart rate } \\ \text { IIAT } & \text { Incremental intermittent Astrand-type test } \\ \text { ISRT } & \text { Incremental stepwise ramp test } \\ \text { [La }]_{\mathrm{b}} & \text { Blood lactate concentration } \\ \text { RER } & \text { Respiratory exchange ratio } \\ S & \text { Ramp slope } \\ t & \text { Time } \\ T & \text { Time to exhaustion } \\ T_{\mathrm{S}} & \text { Step duration } \\ \dot{V}_{\text {Emax }} & \text { Maximal expired ventilation } \\ \dot{V} \mathrm{O}_{2} & \text { Oxygen consumption } \\ \dot{V}_{2} & \text { Maximal oxygen consumption } \\ W^{\prime} & \text { Morton's model constant equal to the work } \\ & \text { carried out to sustain the power above critical } \\ W_{\mathrm{M}} & \text { power } \\ \dot{w} & \text { Total mechanical work } \\ \dot{w}_{\text {max }} & \text { Power } \\ \dot{w}_{\text {peak }} & \text { Maximal aerobic mechanical power } \\ & \text { Peak power of a ramp test }\end{array}$




\section{Introduction}

The maximal oxygen consumption $\left(\dot{V} \mathrm{O}_{2 \max }\right)$ is "an important determinant of the endurance performance, which represents a true parametric measure of cardiorespiratory capacity" (Levine 2008). A variety of incremental exercise test protocols, either continuous or discontinuous, have been proposed in the last decades to measure $\dot{V} \mathrm{O}_{2 \max }$. The most classical protocol for $\dot{V} \mathrm{O}_{2 \max }$ testing is the incremental intermittent $̊$ strand-type test (IIAT) (Åstrand et al. 2003). With IIAT $\dot{V} \mathrm{O}_{2 \max }$ was identified as the plateau attained by the relationship between $\mathrm{O}_{2}$ consumption $\left(\dot{V} \mathrm{O}_{2}\right)$ and power, whence the concept of maximal aerobic mechanical power $\left(\dot{w}_{\max }\right)$ was defined as the power at which the plateau was attained (Åstrand et al. 2003; di Prampero 1981; Howley et al. 1995; Taylor et al. 1955). According to di Prampero (1981), $\dot{w}_{\max }$ represents the power that can be sustained by the active muscle mass with a rate of energy expenditure equivalent to $\dot{V} \mathrm{O}_{2 \max }$. Subsequently, the introduction of commercial breath-by-breath metabolic carts (Myers and Bellin 2000) and the development of electro-magnetically braked cycle ergometers lead to the replacement of the IIAT by the incremental stepwise ramp tests (ISRT). ISRT provide the same $\dot{V} \mathrm{O}_{2 \max }$ values as the IIAT (Duncan et al. 1997; Maksud and Coutts 1971; McArdle et al. 1973), but higher peak powers at the end of the tests $\left(\dot{w}_{\text {peak }}\right)$ the greater is the mean slope of the ramp (Amann et al. 2004; Fairshter et al. 1983; Morton et al. 1997; Zhang et al. 1991).

On this basis, Whipp (1994) proposed a theoretical analysis of $\dot{w}_{\text {peak }}$, whereby he assumed that, if one considers ISRT protocols with fixed power increments between two successive steps and variable step duration, there must be an inverse relationship between $\dot{w}_{\text {peak }}$ and step duration, with asymptote equal to $\dot{w}_{\max }$. Starting from these concepts, Morton (2011) proposed a more detailed model that included critical power (CP) and anaerobic capacity as constants and the mean ramp slope $(S)$ as variable. He validated his model by means of data taken from a previous study with continuous linear increases in $S$ (Morton et al. 1997). Yet most of the ISRT do not foresee continuous linear power increments with time (i.e. power increases at each second) but stepwise power increments, in which power is increased by a given amount at a given fixed time, which may vary from 15 to $300 \mathrm{~s}$. We do not know yet whether and how the data obtained with such a procedure fit into Morton's model. Our hypothesis is that $\dot{w}_{\text {peak }}$ depends only on $S$, and therefore that Morton's model of ramp tests has a general validity, independent of the type of ISRT applied. If this is so, Morton's model should account for differences in $\dot{w}_{\text {peak }}$ obtained with both continuous ramps and ramps characterised by stepwise power increments. The aim of this study was to test this hypothesis by validating
Morton's model of $\dot{w}_{\text {peak }}$ with a series of ISRT tests, in which the power increase was kept invariant and $S$ was varied by changing only the step duration.

\section{Methods}

Subjects

Sixteen healthy, moderately active male subjects, all nonsmokers, volunteered for this study. The subjects' anthropometric characteristics, which were determined on the first experimental session, were as follows (mean $\pm \mathrm{SD}$ ): age $22 \pm 1.7$ years, body mass $73.5 \pm 8.2 \mathrm{~kg}$, height $1.78 \pm 0.4 \mathrm{~m}$. They were asked not to train and to abstain from alcohol on the $24 \mathrm{~h}$ before each experiment, and to have a light meal without coffee intake at least $2 \mathrm{~h}$ before reporting to the laboratory. They were informed about the aims, the procedures and risks associated with the tests and they signed an informed consent form. The study conformed to the Declaration of Helsinki and was approved by the local ethical committee.

Experimental design and methods

Six ISRT protocols and one IIAT protocol were administered in random order. All tests were performed on a cycle ergometer (Ergometrics er800S, Ergoline, Jaeger, Germany) to volitional exhaustion. Successive tests were separated by at least $72 \mathrm{~h}$. The entire protocol was completed within 1 month, during which subjects were instructed to maintain constant the training workload and to refrain from competition. Subjects were asked to pedal at a frequency between 60 and $90 \mathrm{rpm}$. Each of them maintained his own pedalling frequency invariant by visual feedback and used the same pedalling frequency in all tests.

Respiratory gas flows and ventilation were continuously measured breath-by-breath, at the mouth, using a metabolic unit (Quark $b^{2}$, Cosmed, Italy), consisting of a Zirconium Oxygen analyser, an infrared $\mathrm{CO}_{2}$ meter and a turbine flowmeter. The gas analysers were calibrated with ambient air and with a mixture of known gases $\left(\mathrm{O}_{2} 16 \%, \mathrm{CO}_{2} 5 \%\right.$, $\mathrm{N}_{2}$ as balance), and the turbine by means of a 3-1 syringe. Beat-by-beat heart rate (HR) was continuously monitored by cardiotachography (Polar RS $800 \mathrm{CX}$, Polar, Finland). Blood lactate concentration ([La $\left.]_{b}\right)$ was measured on $10 \mu \mathrm{l}$ blood samples taken from an earlobe, by an electro-enzymatic method (Biosen C_line, EKF Diagnostic, Germany).

\section{ISRT protocols}

After $3 \mathrm{~min}$ at rest, the subjects pedalled at $50 \mathrm{~W}$ for $5 \mathrm{~min}$ as warm-up. Then the workload was progressively increased 
in a ladder-like way by $25 \mathrm{~W}$ step, occurring at the end of the selected step duration. As indeed, the six ISRT protocols differed among them only for the step duration, which was: $15,30,60,90,120$ and $180 \mathrm{~s}$. Subjects were encouraged verbally to carry the tests on until exhaustion. $\dot{V} \mathrm{O}_{2}$, expired ventilation and $\mathrm{HR}$ were averaged in the last $10 \mathrm{~s}$ of each workload for all ramps, including those with step duration of 120 and $180 \mathrm{~s}$, after demonstration that the 10-s, 20-s and the 30 -s average provided the same $\dot{V} \mathrm{O}_{2}$ values. The highest $\dot{V} \mathrm{O}_{2}$ value was retained as the individual $\dot{V} \mathrm{O}_{2 \max }$, and the corresponding workload as the individual $\dot{w}_{\text {peak }}$. Blood samples for $[\mathrm{La}]_{\mathrm{b}}$ determination were taken at rest and at 1,3 and 5 min during recovery, in order to assess the peak $[\mathrm{La}]_{\mathrm{b}}$ at the end of the test (di Prampero 1981).

\section{IIAT protocol}

After $3 \mathrm{~min}$ at rest, the first workload was set at $50 \mathrm{~W}$. Each successive load was $50 \mathrm{~W}$ higher than the immediately preceding one. This step increase in power was reduced to $25 \mathrm{~W}$, as the expected maximal $\mathrm{HR}$ was approached. Each workload lasted $5 \mathrm{~min}$. Successive workloads were separated by 6 min recovery intervals. The test was terminated at subject's voluntary exhaustion.

$\dot{V} \mathrm{O}_{2}$, expired ventilation and $\mathrm{HR}$ were obtained as the mean value over the 5 th $\mathrm{min}$ of each workload. Individual $\dot{V} \mathrm{O}_{2 \text { max }}$ was established from the plateau in the $\dot{V} \mathrm{O}_{2}$ versus power relationship. In case of absence of a plateau, the highest measured $\dot{V} \mathrm{O}_{2}$ was retained as $\dot{V} \mathrm{O}_{2 \max }$ if at least two of the following conditions were met: (i) a lack of increase in HR between successive workloads; (ii) respiratory exchange ratio (RER) value $\geq 1.1$; (iii) $[\mathrm{La}]_{\mathrm{b}}$ value higher than $10 \mathrm{mM}$ at maximal exercise.

$\dot{W}_{\max }$ was determined as the power at the intersection between the $\dot{V} \mathrm{O}_{2}$ plateau and the line describing the relationship between $\dot{V} \mathrm{O}_{2}$ and power. In absence of a plateau, the power corresponding to the highest measured $\dot{V} \mathrm{O}_{2}$ was retained. The $\dot{w}_{\max }$ values provided by the IIAT were used to validate the predictions of Whipp's model (see below).

\section{Morton's model}

In Morton's model (Morton 1994, 2011), the power $(\dot{w})$ in an ISRT is admitted to increase continuously with time $(t)$ at a constant rate, so that there is a linear relationship between $\dot{w}$ and $t$ whose angular coefficient is the ramp slope $(S)$ :

$\dot{w}=S \cdot t$

The total mechanical work performed $\left(W_{\mathrm{M}}\right)$ is equal to the triangular area under the $\dot{w}$ versus $t$ line (see Fig. 1), which is equal to:
$W_{\mathrm{M}}=\int_{0}^{T} \dot{w} \mathrm{~d} t=\frac{1}{2} T \cdot \dot{w}_{\text {peak }}=\frac{1}{2} S \cdot T^{2}$

where $T$ is the time to exhaustion. Eq. 2 has the following geometric solution:

$$
\begin{aligned}
\frac{1}{2} T \cdot \dot{w}_{\text {peak }}= & \frac{1}{2} \mathrm{CP}^{2} \cdot S^{-1}+\mathrm{CP} \cdot\left(T-\mathrm{CP} \cdot S^{-1}\right) \\
& +\frac{1}{2}\left(\dot{w}_{\text {peak }}-\mathrm{CP}\right)\left(T-\mathrm{CP} \cdot S^{-1}\right)
\end{aligned}
$$

where CP is the critical power. Each of the three terms of the right branch of Eq. 3 represents a fraction of $W_{\mathrm{M}}$, indicated in Fig. 1 with Greek letters $\alpha, \beta$ and $\gamma$, respectively. The third of these terms ( $\gamma$ in Fig. 1) represents the amount of work carried out to sustain the power above $\mathrm{CP}$, which Morton set equal to the anaerobic work capacity ( $W \prime$ in his notation), i.e. the amount of mechanical work carried out with anaerobic energy sources. In fact the metabolic energy supporting $W /$ consists of a mixture of aerobic and anaerobic energy sources (see di Prampero 1981 for a discussion of this issue). A simplified version of Eq. 3 can be obtained by summing the terms corresponding to areas $\alpha$ and $\beta$ (Fig. 1), and inserting $W /$ :

$\frac{1}{2} T \cdot \dot{w}_{\text {peak }}=\mathrm{CP} \cdot T-\frac{1}{2} \mathrm{CP}^{2} \cdot S^{-1}+W \prime$

whose trigonometric solution for $T$ is given by (Morton 1994):

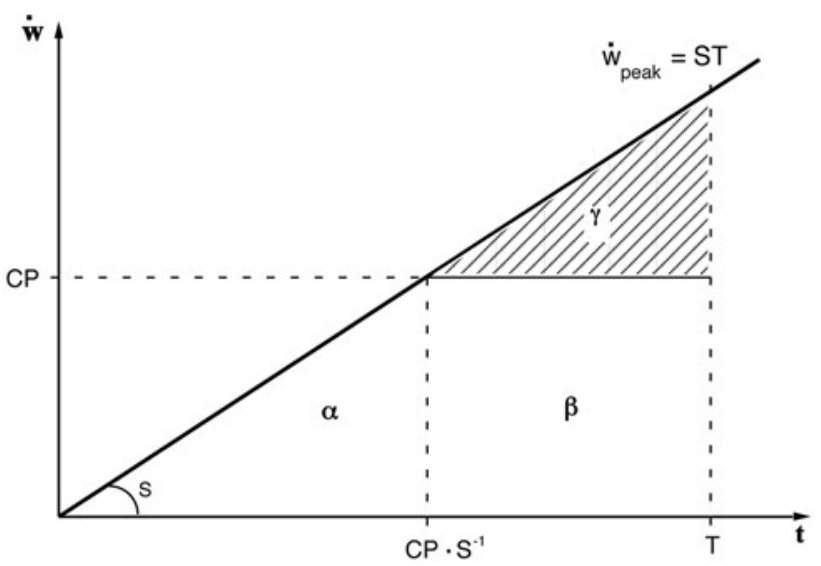

Fig. 1 Geometric representation of Eq. 3. The linear relationship between power $(\dot{w})$ and time $(t)$ is shown, which angular coefficient coincides with the ramp slope $(S)$. The straight bold line represents the increment of power. CP indicates the critical power, and $T$ the time to exhaustion. The area of triangle $\alpha$ is equal to $\frac{1}{2} \mathrm{CP}^{2} \cdot S^{-1}$ (first term of right branch of Eq. 3, while that of rectangle $\beta$ is equal to $\mathrm{CP} \cdot\left(T-\mathrm{CP} \cdot S^{-1}\right)$ (second term of right branch of Eq. 3), and that of triangle $\gamma$ is equal to $\frac{1}{2}\left(\dot{w}_{\text {peak }}-\mathrm{CP}\right)\left(T-\mathrm{CP} \cdot S^{-1}\right)$. Morton (2011) indentified $\gamma$ area as the anaerobic work capacity ( $W^{\prime}$ in his notation, striped area in this figure). The sum of areas $\alpha+\beta+\gamma$ corresponds to the total mechanical work performed $\left(\frac{1}{2} \cdot \dot{w}_{\text {peak }} \cdot T\right)$ 
$T=\mathrm{CP} \cdot S^{-1}+\sqrt{\frac{2 W \prime}{S}}$

If we then multiply Eq. 5 by $S$, we get:

$$
S \cdot T=\dot{w}_{\text {peak }}=\mathrm{CP}+\sqrt{2 W / S}
$$

Eq. 6 tells that, if we plot $\dot{w}_{\text {peak }}$ as a function of $\sqrt{S}$ we obtain linear relationships with slope equal to $\sqrt{2 W \prime}$ and $\mathrm{y}$-intercept equal to $\mathrm{CP}$. This equation was tested by using the results of the six ISRT, with $S$ corresponding to the mean ramp slope.

Whipp's model

A simpler, concurrent model for the analysis of $\dot{w}_{\text {peak }}$ was previously proposed by Whipp (1994) for ISRT protocols characterised by discrete ramps with the steps of different duration. This model predicted an inverse relationship between $\dot{w}_{\text {peak }}$ and step duration, described by a translated equilateral hyperbola of this form:

$T_{\mathrm{S}} \cdot\left(\dot{w}_{\text {peak }}-b\right)=a$

where $T_{\mathrm{S}}$ is the step duration. According to Whipp (1994), constant $b$ is equivalent to $\dot{w}_{\max }$ and constant $a$ to the anaerobic work. Thus, Eq. 7 can be linearized as:

$\dot{w}_{\text {peak }}=\frac{a}{T_{\mathrm{S}}}+b$

\section{Statistics}

Values are reported as mean and standard deviation (SD). A one-way ANOVA for repeated measurements was used to compare results from the various protocols. A Tukey post hoc test was then applied to locate significant differences. The level of significance was set at $P<0.05$. Linear regressions were calculated by means of least square methods. The statistical package Prism 6 (Version 6.0b, GraphPad Software Inc., La Jolla, CA, USA) was used.

\section{Results}

The mean values of all measured variables for each maximal incremental test are reported in Table 1. No significant difference appeared for $\dot{V} \mathrm{O}_{2 \max }$ and maximal expired ventilation $\left(\dot{V}_{E_{\max }}\right)$ among the protocols. In contrast, the ISRT $\dot{w}_{\text {peak }}$ was lower the longer the step duration, with differences being significant among all step durations. Moreover, all $\dot{w}_{\text {peak }}$ obtained in the various ISRT were significantly different from the $\dot{w}_{\max }$ reached at the end of IIAT. Consistently, maximal RER slightly decreased with increasing the ramp step duration. At the end of the test, no differences were observed for HR, except for the two shortest protocols (15 and $30 \mathrm{~s}$ ISRT), during which a significantly lower HR was reached compared with the two longest tests (180 s ISRT and IIAT). No differences were found for the peak $[\mathrm{La}]_{\mathrm{b}}$ values among the various ISRT. However, a few peak $[\mathrm{La}]_{b}$ values were significantly different from the peak [La $]_{b}$ of IIAT, for a few low values in ISRT.

Only the six ISRT $\dot{w}_{\text {peak }}$ values were analysed with respect to Morton's model in Fig. 2, where $\dot{w}_{\text {peak }}$ was plotted as a function of $\sqrt{S}$ (see Eq. 6). From individual linear regression analysis, mean $\mathrm{CP}$ turned out equal to $198.08 \pm 37.46 \mathrm{~W}$, i.e. $74.2 \%$ of assessed $\dot{w}_{\max }$, and $W^{\prime}$ resulted equal to $16.82 \pm 5.69 \mathrm{~kJ}$. The mean value of the individual regression coefficients was $0.981 \pm 0.016$. The overall regression line on all individual data, reported in Fig. 2 , is described by the following equation: $\dot{w}_{\text {peak }}=$ $180.95 \sqrt{S}+198.04$; the correlation coefficient is equal to 0.819 , reflecting inter-subject variability.

On Fig. 3 we reported a theoretical solution of Eq. 8, obtained by setting $a=W \prime$ (from Fig. 2) and $b=\dot{w}_{\max }$ (from Table 1, IIAT column). The present experimental data were also reported on the same figure. The equation provided by linear regression analysis on the latter data was $y=2612 x+263.5, r=0.976$, indicative of $a \dot{w}_{\max }$ of $264 \mathrm{~W}$

\section{Discussion}

The $\dot{V} \mathrm{O}_{2 \max }$ observed in the present study was the same in all performed tests, independent of the protocol used and, thus, independent of the step duration applied on an ISRT, in agreement with previous reports (Amann et al. 2004; Bentley et al. 2007; Bishop et al. 1998; Duncan et al. 1997; Hughson et al. 2000; Maksud and Coutts 1971; McArdle et al. 1973; McNaughton et al. 2005; Morton et al. 1997). As a consequence, both ISRT and IIAT can be conveniently used to determine $\dot{V} \mathrm{O}_{2 \max }$. This result is coherent with the conclusions already arrived at by Howley et al. (1995) after having compared $\dot{V} \mathrm{O}_{2 \max }$ values obtained with continuous or discontinuous exercise testing protocols, from different sources in the literature. In spite of this, the $\dot{w}_{\text {peak }}$ values attained in ISRT were significantly different among them and from the $\dot{w}_{\max }$ attained in IIAT.

Morton et al. (1997) using continuous linear ramp increments of different slope, obtained similar results. Figure 2 shows excellent fitting of present results with Morton's model. Since our data were obtained using stepwise power increments, with duration of constant-load steps ranging from 15 to $180 \mathrm{~s}$, the present study extends the domain of utilisation of Morton's model ideally to any type of ramp protocol. If this is so, only the mean ramp 

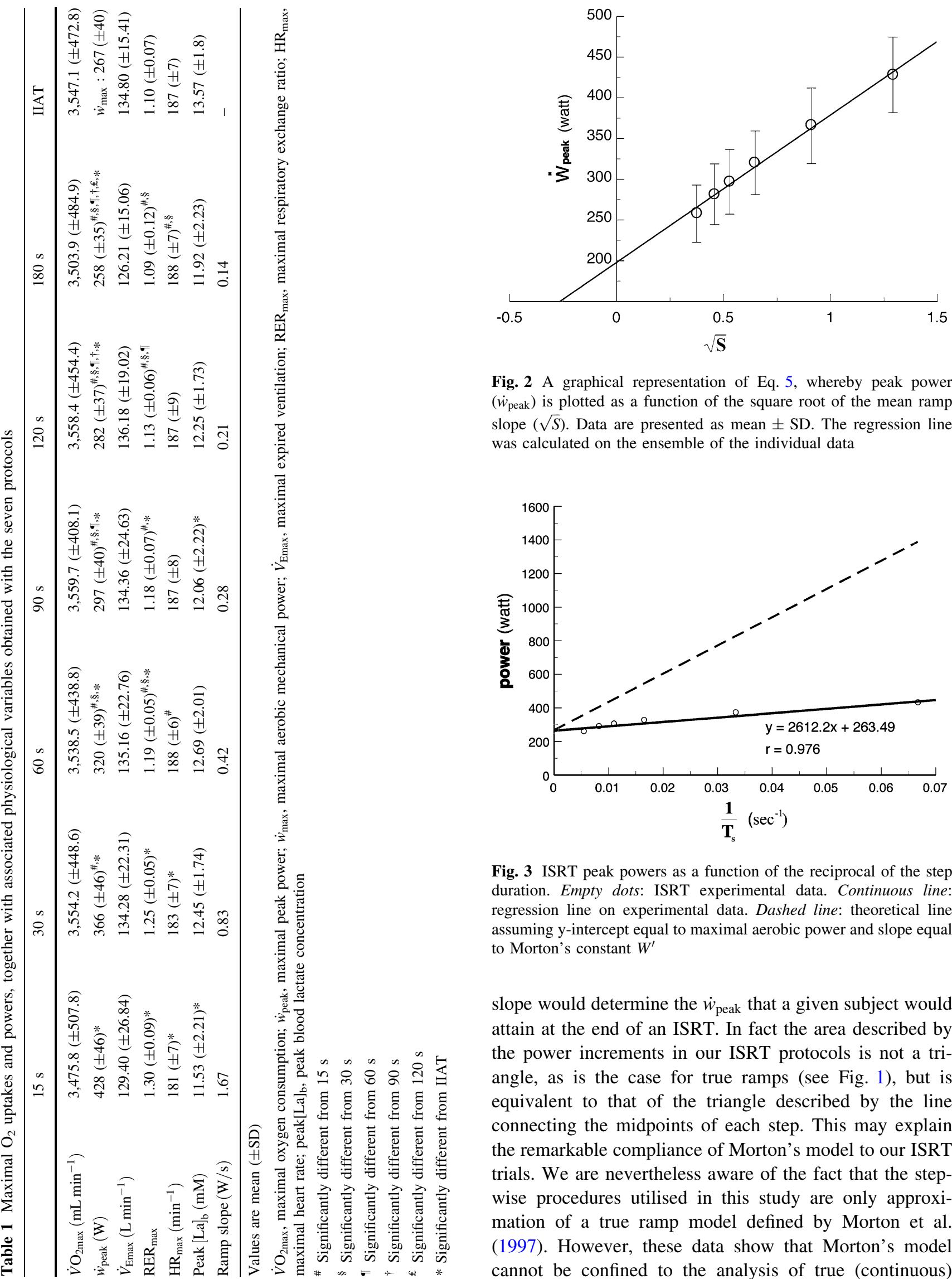

Fig. 2 A graphical representation of Eq. 5, whereby peak power $\left(\dot{w}_{\text {peak }}\right)$ is plotted as a function of the square root of the mean ramp slope $(\sqrt{S})$. Data are presented as mean \pm SD. The regression line was calculated on the ensemble of the individual data

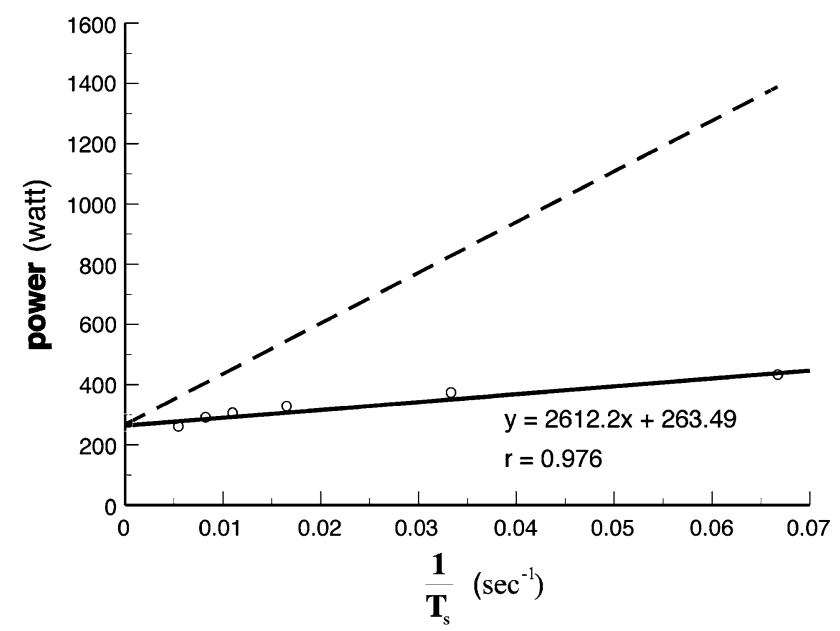

Fig. 3 ISRT peak powers as a function of the reciprocal of the step duration. Empty dots: ISRT experimental data. Continuous line: regression line on experimental data. Dashed line: theoretical line assuming y-intercept equal to maximal aerobic power and slope equal to Morton's constant $W^{\prime}$

slope would determine the $\dot{w}_{\text {peak }}$ that a given subject would attain at the end of an ISRT. In fact the area described by the power increments in our ISRT protocols is not a triangle, as is the case for true ramps (see Fig. 1), but is equivalent to that of the triangle described by the line connecting the midpoints of each step. This may explain the remarkable compliance of Morton's model to our ISRT trials. We are nevertheless aware of the fact that the stepwise procedures utilised in this study are only approximation of a true ramp model defined by Morton et al. (1997). However, these data show that Morton's model cannot be confined to the analysis of true (continuous) 
ramp protocols but its use can be expanded to include all protocols in which there is no interruption between successive steps. A further expansion would result from an inclusion also of Morton and Billat (2004) model of intermittent protocols, starting from the consideration that ISRT can be approximated to intermittent tests with recovery between successive steps equal to $0 \mathrm{~s}$.

Recently, Morton (Morton 2011) expanded the original CP model (Monod and Scherrer 1965) to include an analysis of maximal ramp tests by means of a new model. Fig. 2 presents a linearized analytical form of that model, based on Eq. 5. The line's slope, according to this equation, is equal to $\sqrt{2 W}$. Morton (2011) stated that $W /$ corresponds to the subjects' anaerobic capacity, yet we think this is an oversimplification. An analysis of the energy balance at exercise intensity between $\mathrm{CP}$ and $\dot{w}_{\text {peak }}$ shows that $W$ I includes at least two terms: (i) the energy derived from anaerobic lactic energy sources, and (ii) the energy provided by the further increase in $\dot{V} \mathrm{O}_{2}$ at powers above CP. As a consequence, contrary to Morton's prediction, W' is larger than anaerobic capacity.

On the other hand, the predictions resulting from application of Whipp's model (Fig. 3), indicated that (i) the y-intercept of $264 \mathrm{~W}$ was indeed very close to the measured $\dot{w}_{\text {max }}$ that we obtained during the IIAT (267 W, see Table 1), as predicted by Whipp (1994); whereas (ii) the regression slope $a$, which corresponded to $2.61 \mathrm{~kJ}$, was about $1 / 7$ of $W /$. We suggest that the latter discrepancy may depend on the different meaning of $a$ and $W \prime$. Whereas $a$ is the energy from anaerobic sources used to sustain powers above $\dot{w}_{\max }, W /$ which includes $a$, is the energy (aerobic and anaerobic) sustaining all the work carried out above CP. We can therefore state that Eqs. 5 and 7 are equally good tools for the description of the $\dot{w}_{\text {peak }}$ attained in ISRT.

The solutions of Eqs. 6 and 8 for $\dot{w}_{\text {peak }}$ must be equivalent, so that they can be combined as follows:

$\frac{a}{T_{\mathrm{S}}}+\dot{w}_{\max }=\mathrm{CP}+\sqrt{2 W^{\prime} S}$

Rearrangement of Eq. 9 provides:

$\sqrt{2 W / S}=\frac{a}{T_{\mathrm{S}}}+\left(\dot{w}_{\max }-\mathrm{CP}\right)$

Equation 10 tells that $\sqrt{2 W^{\prime} S}$ varies linearly with $\frac{1}{T_{\mathrm{S}}}$, with y-intercept equal to $\left(\dot{w}_{\max }-\mathrm{CP}\right)$ and slope equal to $a$. This implies that (i) the difference between $\dot{w}_{\max }$ and CP is a constant that is independent of anaerobic capacity, step duration and ramp slope; (ii) $\dot{w}_{\max }$ and $\mathrm{CP}$ are bound to vary together, and by the same absolute amount; and (iii) their ratio becomes higher the higher is $\dot{w}_{\max }$, and therefore there is no fix $\mathrm{CP} / \dot{w}_{\max }$ ratio. In practice, Eq. 10 explains why (i) CP is a higher fraction of $\dot{w}_{\max }$ in athletes with elevated $\dot{V} \mathrm{O}_{2 \max }$ (Heubert et al. 2005) than in subjects with low $\dot{V} \mathrm{O}_{2 \text { max }}$, as the present ones; and (ii) the $\mathrm{CP} / \dot{w}_{\text {max }}$ ratio may vary with aerobic training (Heubert et al. 2003), and perhaps, we speculate, may differ according to muscle fibre composition. Since CP is strongly related to the so-called anaerobic threshold, a concept widely used in sport science, Eq. 10 also explains why intense aerobic training improves both $\dot{w}_{\max }$ and the anaerobic threshold, or the sustainable fraction of $\dot{V} \mathrm{O}_{2 \max }$ (di Prampero 1986; Tam et al. 2012).

\section{Conclusions}

In conclusion, the quantitative analysis illustrated in this study demonstrated that Morton's model well describes the evolution of $\dot{w}_{\text {peak }}$ with any type of ramp, underlying once more that its magnitude is protocol-dependent. Moreover, a practical consideration emerges: performing a series of multiple (minimum three) ISRTs, varying in slope but not in power increment, allows the computation of the two other important physiological parameters highlighted in this study: $\dot{w}_{\max }$ and CP. The application of our analysis ensures an adequate methodology to correctly determine these parameters that are bound to vary together, by the same absolute amount, so that their ratio will result higher the higher is $\dot{V} \mathrm{O}_{2 \max }$. The simultaneous knowledge of $\dot{V} \mathrm{O}_{2 \max }, \dot{w}_{\max }$, and $\mathrm{CP}$ improves our ability of defining correct training programmes.

Acknowledgments The authors thank all the volunteers who participated in this study. This research was supported by the Swiss National Science Foundation Grant 32003B_127620 to G. Ferretti. The authors declare that they have no conflict of interest.

\section{References}

Amann M, Subudhi A, Foster C (2004) Influence of testing protocol on ventilatory thresholds and cycling performance. Med Sci Sports Exerc 36:613-622

Åstrand PO, Rodahl K, Dahl HA, Strømme SB (2003) Textbook of work physiology. Physiological bases of exercise, fourth ed. Human Kinetics, Champaign

Bentley DJ, Newell J, Bishop D (2007) Incremental exercise test design and analysis. Implications for performance diagnostics in endurance athletes. Sports Med 37:575-586

Bishop D, Jekins DG, Mackinnon LT (1998) The effect of stage duration on the calculation of peak $\mathrm{VO}_{2}$ during cycle ergometry. J Sci Med Sport 1:171-178

di Prampero PE (1981) Energetics of muscular exercise. Rev Physiol Bioch P 89:143-222

di Prampero PE (1986) The energy cost of human locomotion on land and in water. Int J Sports Med 7:55-72

Duncan GE, Howley ET, Johnson BN (1997) Applicability of $\mathrm{VO}_{2 \max }$ criteria: discontinuous versus continuous protocols. Med Sci Sports Exerc 29:273-278 
Fairshter RD, Walters J, Salness K, Fox M, Minh VD, Wilson AF (1983) A comparison of incremental exercise tests during cycle and treadmill ergometry. Med Sci Sports Exerc 15:549-554

Heubert R, Bocquet V, Koralsztein JP, Billat VL (2003) Effets de 4 semaines d'entraînement sur le temps limite à $\mathrm{VO}_{2 \max }$. Can $\mathrm{J}$ Appl Physiol 28:717-736

Heubert RAP, Billat VL, Chasseaing P, Bocquet V, Morton RH, Koralsztein JP, di Prampero PE (2005) Effects of a previous sprint on the parameters of the work-time to exhaustion relationship in high intensity cycling. Int $\mathrm{J}$ Sport Med 26:583-592

Howley ET, Bassett DR Jr, Welch HG (1995) Criteria for maximal oxygen uptake: review and commentary. Med Sci Sports Exerc 27:1292-1301

Hughson RL, O'Leary DD, Betik AC, Hebestreit H (2000) Kinetics of oxygen uptake at the onset of exercise near or above peak oxygen uptake. J Appl Physiol 88:1812-1819

Levine BD (2008) $\mathrm{VO}_{2 \max }$ : what do we know, and what do we still need to know? J Physiol 586:25-34

Maksud MG, Coutts KD (1971) Comparison of a continuous and discontinuous graded treadmill test for maximal oxygen uptake. Med Sci Sports Exerc 3:63-65

McArdle WD, Katch FI, Pechar GS (1973) Comparison of continuous and discontinuous treadmill and bicycle tests for max $\mathrm{VO}_{2}$. Med Sci Sports Exerc 3:156-160

McNaughton LR, Roberts S, Bentley BJ (2005) Predicting performance in a short distance cycling time trial: effects of incremental exercise test design. J Strength Cond Res 20:157-161

Monod H, Scherrer J (1965) The work capacity of a synergic muscular group. Ergonomics 8:329-338

Morton RH (1994) Critical power test for ramp exercise. Eur J Appl Physiol 69:435-438

Morton RH (2011) Why peak power is higher at the end of steeper ramps: an explanation based on the "critical power" concept. J Sport Sci 29:307-309

Morton RH, Billat LV (2004) The critical power model for intermittent exercise. Eur J Appl Physiol 91:303-307

Morton RH, Green S, Bishop D, Jekins DG (1997) Ramp and constant power trials produce equivalent critical power estimates. Med Sci Sports Exerc 29:833-836

Myers J, Bellin D (2000) Ramp exercise protocols for clinical and cardiopulmonary exercise testing. Sports Med 30:23-29

Tam E, Rossi H, Moia C, Berardelli C, Rosa G, Capelli C, Ferretti G (2012) Energetics of running in top-level marathon runners from Kenya. Eur J Appl Physiol 112:3797-3806

Taylor HL, Buskirk E, Henschel A (1955) Maximal oxygen uptake as an objective measure of cardiorespiratory performance. J Appl Physiol 8:73-80

Whipp BJ (1994) The bioenergetic and gas exchange basis of exercise testing. Clin Chest Med 15:173-192

Zhang YY, Johnson MC 2nd, Chow N, Wasserman K (1991) Effect of exercise testing protocol on parameters of aerobic function. Med Sci Sports Exerc 23:625-630 